\title{
Transverse Momentum Distributions in proton - proton Collisions at LHC Energies and Tsallis Thermodynamics
}

\author{
M.D. Azmi ${ }^{1}$ and J. Cleymans ${ }^{2}$ \\ ${ }^{1}$ HEP Lab, Department of Physics, Aligarh Muslim University, Aligarh - 202002, India \\ ${ }^{2}$ UCT-CERN Research Centre and Department of Physics, University of Cape Town, \\ Rondebosch 7701, South Africa
}

\begin{abstract}
A detailed study of the transverse momentum distributions of charged particles produced in proton - proton collisions at LHC energies is presented. This is done using a thermodynamically consistent form of the Tsallis distribution. All variables used are thermodynamical and in particular, the temperature, $T$, follows from the standard thermodynamic definition as being the derivative of the energy with respect to the (Tsallis) entropy. The momentum distribution of the final state particles can be described very well by the Tsallis distribution. The values of the parameters are determined from measurements by the ALICE, ATLAS and CMS collaborations and are discussed in detail. In particular, the Tsallis parameter, $q$, is found with consistent values for all the transverse momentum distributions despite large differences in kinematic regions and shows a slight increase with beam energy, reaching a value of 1.15 at $7 \mathrm{TeV}$. It is concluded that the hadronic system created in highenergy $\mathrm{p}$ - $\mathrm{p}$ collisions at mid-rapidity can be seen as obeying Tsallis thermodynamics.
\end{abstract}

PACS numbers: 12.40.Ee,13.75.Cs,13.85.-t,05.70.-a,05.70.-a 


\section{Introduction}

The dynamics of colliding hadrons can be determined from the distribution of the momenta of produced particles. The available range of transverse momenta has expanded considerably with the advent of the Large Hadron Collider (LHC) at CERN. Collider energies up to 7 $\mathrm{TeV}$ are now available in proton - proton collisions and transverse momenta of hundreds of $\mathrm{GeV}$ are of a common occurrence. This helps in testing and applying the relativistic thermodynamics and hydrodynamics in a new energy region. Thermal models are part of the standard set of tools to analyze high-energy collisions and in this paper we investigate a thermodynamically consistent model based on the Tsallis distribution [1, 2]. This leads to a power law distribution which is well suited to describe the transverse momenta measured in $\mathrm{p}$ - $\mathrm{p}$ collisions. The Tsallis distribution was first proposed about twenty-five years ago as a generalization of the usual exponential Boltzmann-Gibbs distribution, and is characterized by three parameters $q, T$ and $V$. Various versions of the Tsallis distribution have been considered in the literature [3, 4, 5, 6, 7, 8, 9]. A form which is suited for describing results in high energy particle physics is used in this paper.

The variable $T$ used here obeys the standard thermodynamic relation [10, 11],

$$
T=\left.\frac{\partial E}{\partial S}\right|_{N, V},
$$

and can, therefore, be referred as temperature. However, since the entropy used in Eq. (1) is the Tsallis entropy [1] and not the standard entropy, we will call the variable defined in Eq. (1) as q-temperature. For a similar reason the volume $V$ can be obtained from thermodynamic relations as,

$$
V=\left.\frac{\partial H}{\partial P}\right|_{N, S},
$$

with $H=E+P V$ is the standard definition of the enthalpy and could be called q-volume as it is obtained at fixed Tsallis entropy but we refrain from doing so and refer to it as volume but note that it is not necessarily related to a volume deduced from other models, say HBT calculations.

A lot of interest has developed recently [12, 13, 14, 15, 16] in the Tsallis distribution and it has been used previously to describe the transverse momentum data of the charged particles produced in proton - proton collisions at RHIC and LHC energies [17, 18, 19, 20, 21, 22, 23, 24]. A comparative study, which has not been done before, of the Tsallis fits to the transverse momentum distributions of the charged particles produced in proton - proton collisions measured by ALICE, ATLAS and CMS collaborations is presented here. The parameters used in the Tsallis fit are studied at different energies and under different kinematical conditions of the data collection. The parameters are measured at various multiplicities. The fit results the values of Tsallis parameter, $q$, in the range 1.1 to 1.15 for all the measured distributions and found to be consistent in all the conditions and at all energies. The $T$ and $R$ parameters show dependence on the multiplicity and on particle yields too. The results presented here confirm that the hadronic system created in high-energy $\mathrm{p}$ - $\mathrm{p}$ collisions obeys Tsallis thermodynamics. 


\section{Tsallis Distribution}

The transverse momentum distribution in heavy-ion collisions is often described by a combination of transverse flow and a thermodynamical statistical distribution. In $p-p$ collisions with the Tsallis distribution such a superposition is not needed and very good fits can be obtained.

In the framework of Tsallis statistics [1, 8, 12, 10, 11] integrals over

$$
f=\left[1+(q-1) \frac{E-\mu}{T}\right]^{-\frac{1}{q-1}}
$$

give the entropy, $S$, the particle number, $N$, the energy density, $\varepsilon$, and the pressure, $P$.

Using the function

$$
\ln _{q}(x) \equiv \frac{x^{1-q}-1}{1-q},
$$

often referred to as q-logarithm, it can be shown[11] that the relevant thermodynamic quantities are given by:

$$
\begin{aligned}
& S=-g V \int \frac{d^{3} p}{(2 \pi)^{3}}\left[f^{q} \ln _{q} f-f\right], \\
& N=g V \int \frac{d^{3} p}{(2 \pi)^{3}} f^{q}, \\
& \varepsilon=g \int \frac{d^{3} p}{(2 \pi)^{3}} E f^{q}, \\
& P=g \int \frac{d^{3} p}{(2 \pi)^{3}} \frac{p^{2}}{3 E} f^{q} .
\end{aligned}
$$

where $V$ is the volume and $g$ is the degeneracy factor.

In order to use the above equations it has to be shown that they satisfy the thermodynamic

consistency conditions. The first and second laws of thermodynamics lead to the following two differential relations [25]:

$$
\begin{aligned}
& d \varepsilon=T d s+\mu d n, \\
& d P=s d T+n d \mu .
\end{aligned}
$$

where, $s=S / V$ and $n=N / V$ are the entropy and particle number densities, respectively.

Thermodynamic consistency requires that the following relations be satisfied:

$$
\begin{aligned}
& T=\left.\frac{\partial \varepsilon}{\partial s}\right|_{n}, \\
& \mu=\left.\frac{\partial \varepsilon}{\partial n}\right|_{s}, \\
& n=\left.\frac{\partial P}{\partial \mu}\right|_{T}, \\
& s=\left.\frac{\partial P}{\partial T}\right|_{\mu} .
\end{aligned}
$$


Eq. (10) in particular shows that the variable $T$ appearing in Eq. (3) indeed can be identified as a thermodynamic temperature. As explained in the introduction we prefer to call it q-temperature as it is based on the Tsallis form of the entropy [1]. It is straightforward to show that these relations are indeed satisfied [11].

It can easily be shown that the following thermodynamic consistency relation is also satisfied:

$$
\varepsilon+P=T s+\mu n
$$

The momentum distribution obtained from Eq. (5) is given by:

$$
\frac{d^{3} N}{d^{3} p}=\frac{g V}{(2 \pi)^{3}}\left[1+(q-1) \frac{E-\mu}{T}\right]^{-q /(q-1)}
$$

or expressed in terms of variables used in high-energy physics, transverse momentum, $p_{T}$, transverse mass, $m_{T}=\sqrt{p_{T}^{2}+m^{2}}$ and rapidity, $y$ :

$$
\frac{d^{2} N}{d p_{T} d y}=g V \frac{p_{T} m_{T} \cosh y}{(2 \pi)^{2}}\left[1+(q-1) \frac{m_{T} \cosh y-\mu}{T}\right]^{-q /(q-1)}
$$

At mid-rapidity, $y=0$, and for zero chemical potential, as is relevant at the LHC, Eq. (16) reduces to the following expression:

$$
\left.\frac{d^{2} N}{d p_{T} d y}\right|_{y=0}=g V \frac{p_{T} m_{T}}{(2 \pi)^{2}}\left[1+(q-1) \frac{m_{T}}{T}\right]^{-q /(q-1)}
$$

It is worth to mention that the parameterization given in Eq. (17) is close to the parameterization used for fitting the data taken at RHIC and LHC experiments [17, 18, 19, 20, 21, 22, 23, 24]. The parameterization used by the RHIC and LHC experiments is given below:

$$
\frac{d^{2} N}{d p_{T} d y}=p_{T} \frac{d N}{d y} \frac{(n-1)(n-2)}{n C\left(n C+m_{0}(n-2)\right)}\left[1+\frac{m_{T}-m_{0}}{n C}\right]^{-n},
$$

where $n, C$ and $m_{0}$ are the fit parameters used in the parameterization.

At mid-rapidity, $y=0$, and for zero chemical potential Eq. (18) shows the same dependence on the transverse momentum as Eq. (17) except for an additional factor $m_{T}$ which is present in Eq. (17) but not in Eq. (18).

\section{Fit details}

The transverse momentum distributions of charged particles produced in $p-p$ collisions at LHC energies were fitted using a sum of three Tsallis distributions. These consist of fits for $\pi^{+}$'s, $K^{+}$'s and protons, $p$. The following expression, at mid-rapidity and $\mu=0$, was used to fit the distributions obtained in various experiments:

$$
\left.\frac{d^{2} N_{c h}}{d p_{T} d y}\right|_{y=0}=2 p_{T} \frac{V}{(2 \pi)^{2}} \sum_{i=1}^{3} g_{i} m_{T, i}\left[1+(q-1) \frac{m_{T, i}}{T}\right]^{\frac{-q}{(q-1)}}
$$

where $i=\pi^{+}, K^{+}, p$. The relative weights between particles were determined by the corresponding degeneracy factors and given by $g_{\pi^{+}}=g_{K^{+}}=1$ and $g_{p}=2$. The factor 2 
on the right hand side takes into account the contributions from antiparticles, $\pi^{-}, K^{-}$and $\bar{p}$.

In some cases the following form of the distribution was used to fit the experimental data sets:

$$
\left.\frac{1}{2 \pi p_{T}} \frac{d^{2} N_{c h}}{d p_{T} d y}\right|_{y=0}=\frac{2 V}{(2 \pi)^{3}} \sum_{i=1}^{3} g_{i} m_{T, i}\left[1+(q-1) \frac{m_{T, i}}{T}\right]^{\frac{-q}{(q-1)}}
$$

The transverse momentum spectra of primary charged particles measured by the ALICE collaboration [20] in INEL $p-p$ collisions at $\sqrt{s}=900 \mathrm{GeV}(|\eta|<0.8)$, normalized to the total number of INEL events, $N_{\text {evt }}$, for three different multiplicity selections $\left(n_{a c c}\right)$ together with the Tsallis fits, Eq. (17), is shown in Fig. 1.

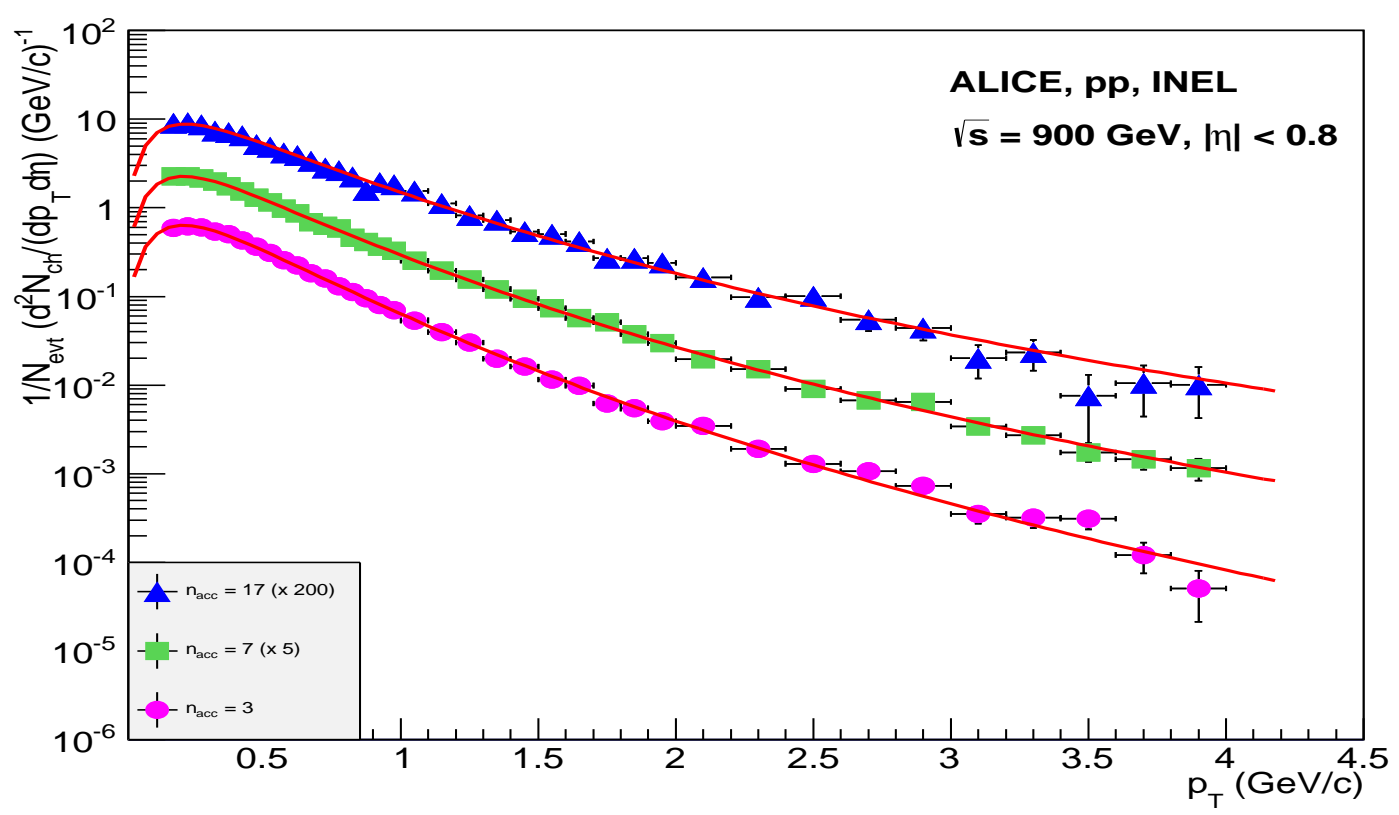

Figure 1. Transverse momentum distributions of charged particles as measured by the ALICE collaboration in $p-p$ collisions at $\sqrt{s}=0.9 \mathrm{TeV}$ fitted with Tsallis distribution.

The charged hadron yields as measured by the CMS collaboration [21, 22] in the range $|\eta|<2.4$ in non-single-diffractive (NSD) events as a function of $p_{T}$ at all three center-of-mass energies, fitted with Tsallis distribution are shown in Fig. 2.

Fig. 3 shows the charged particle multiplicities as a function of transverse momentum measured by the ATLAS collaboration [23] for events with $n_{c h} \geq 1, p_{T}>500 \mathrm{MeV}$ and $|\eta|<2.5$ at $\sqrt{s}=0.9,2.36$ and $7 \mathrm{TeV}$ fitted with Tsallis distribution.

The Tsallis fits for the charged particle multiplicities as a function of transverse momentum measured by the ATLAS collaboration [23] for events with $n_{c h} \geq 2, p_{T}>100$ $\mathrm{MeV}$ and $|\eta|<2.5$ at $\sqrt{s}=0.9$ and $7 \mathrm{TeV}$ are shown in Fig. 4.

Fig. 5 shows the Tsallis fits on the charged particle multiplicities as a function of transverse momentum measured by the ATLAS collaboration [23] for events with $n_{c h} \geq 6$, $p_{T}>500 \mathrm{MeV}$ and $|\eta|<2.5$ at $\sqrt{s}=0.9$ and $7 \mathrm{TeV}$.

Fig. 6 shows the Tsallis fits for the charged particle cross section for three different beam 


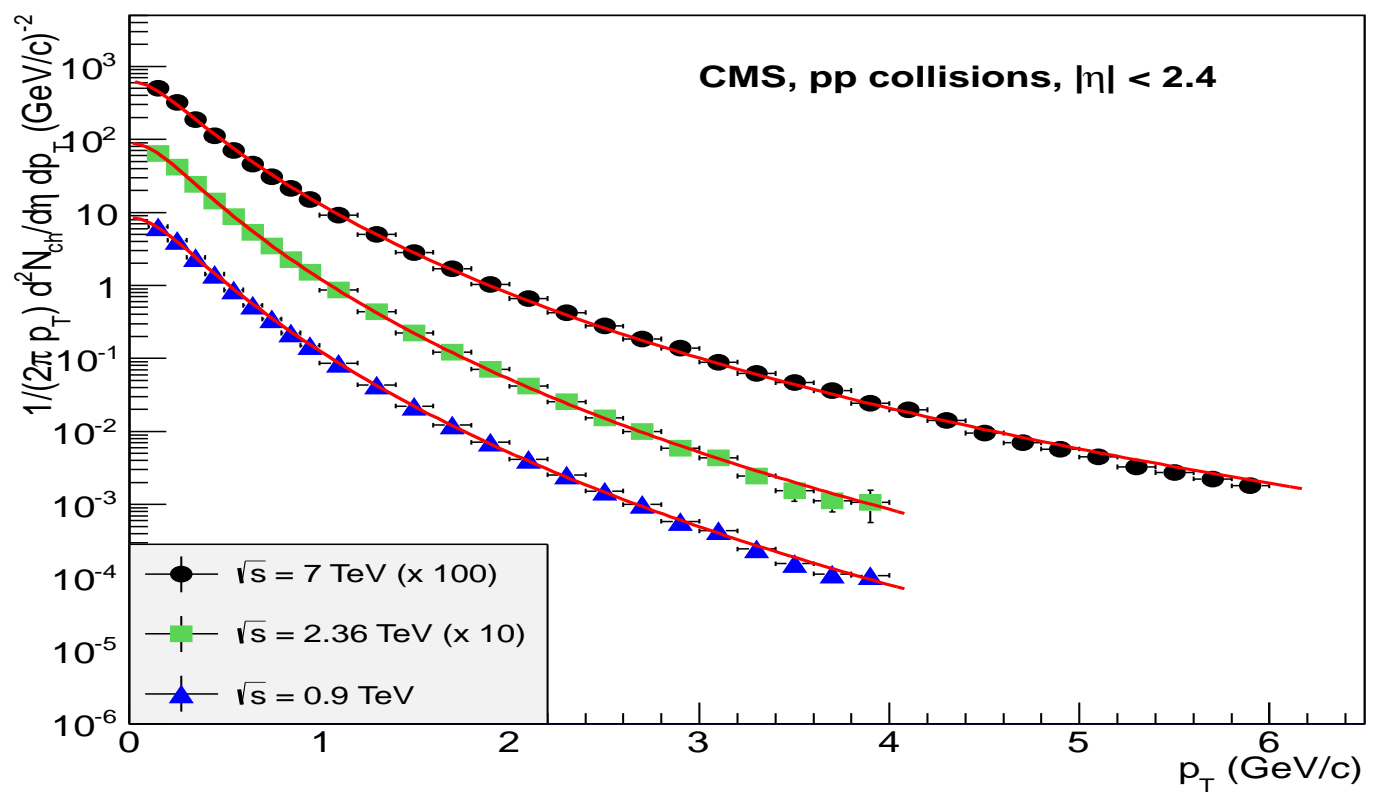

Figure 2. Transverse momentum distributions of charged particles as measured by the CMS collaboration in $p-p$ collisions at $\sqrt{s}=0.9,2.36$ and $7 \mathrm{TeV}$ fitted with Tsallis distribution.

energies, 0.9, 2.76 and $7 \mathrm{TeV}$ measured recently by the ALICE collaboration [24].

In all cases the fits are very good.

\section{Results}

The transverse momentum spectrum of the primary charged particles measured by the ALICE, CMS and ATLAS collaborations and cross section measured by the ALICE collaboration [20, 21, 22, 23, 24] in $p-p$ collisions at LHC energies were fitted using the Tsallis distribution given in Eq. (17).

Though, the kinematical conditions of the data taking of all the three collaborations were different. The Tsallis distribution fits well to the measured $p_{T}$ spectrum as well as the cross section.

The values of the Tsallis parameter, $q$, the q-temperature, $T$, and the radius, $R$, defined as $R \equiv(3 V / 4 \pi)^{1 / 3}$ obtained from the fits of the $p_{T}$ spectrum are shown in Figs. 7, 8 and 9, respectively. The values of the $q$ parameter shows a clear increase with beam energy. The collaborations show consistent compatible results. It is to be noted that the parameter $q$ can be determined with a fairly high precision.

The results for the q-temperature, $T$, shown in Fig. 8 are compatible and show no clear dependence on the beam energy. The only points that are clearly higher are those obtained by the ATLAS collaboration for data with a high number of charged particles $\left(n_{c h} \geq 6\right)$.

The results for the parameter $R$ again show a clear increase with beam energy, which was not seen in a previous analysis [16]. Again the results are compatible. 


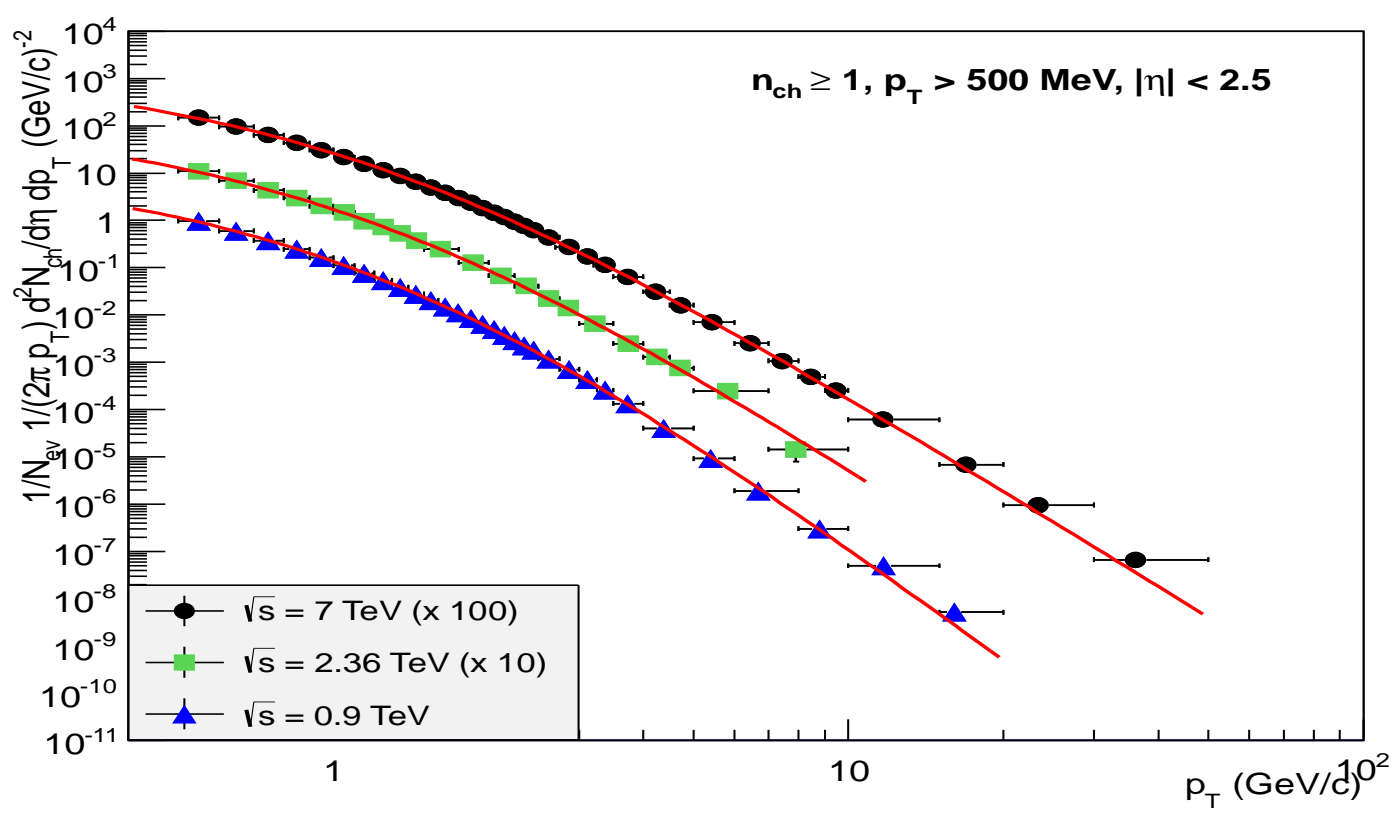

Figure 3. Transverse momentum distributions of charged particles as measured by the ATLAS collaboration at all three center-of-mass energies together with Tsallis fit.

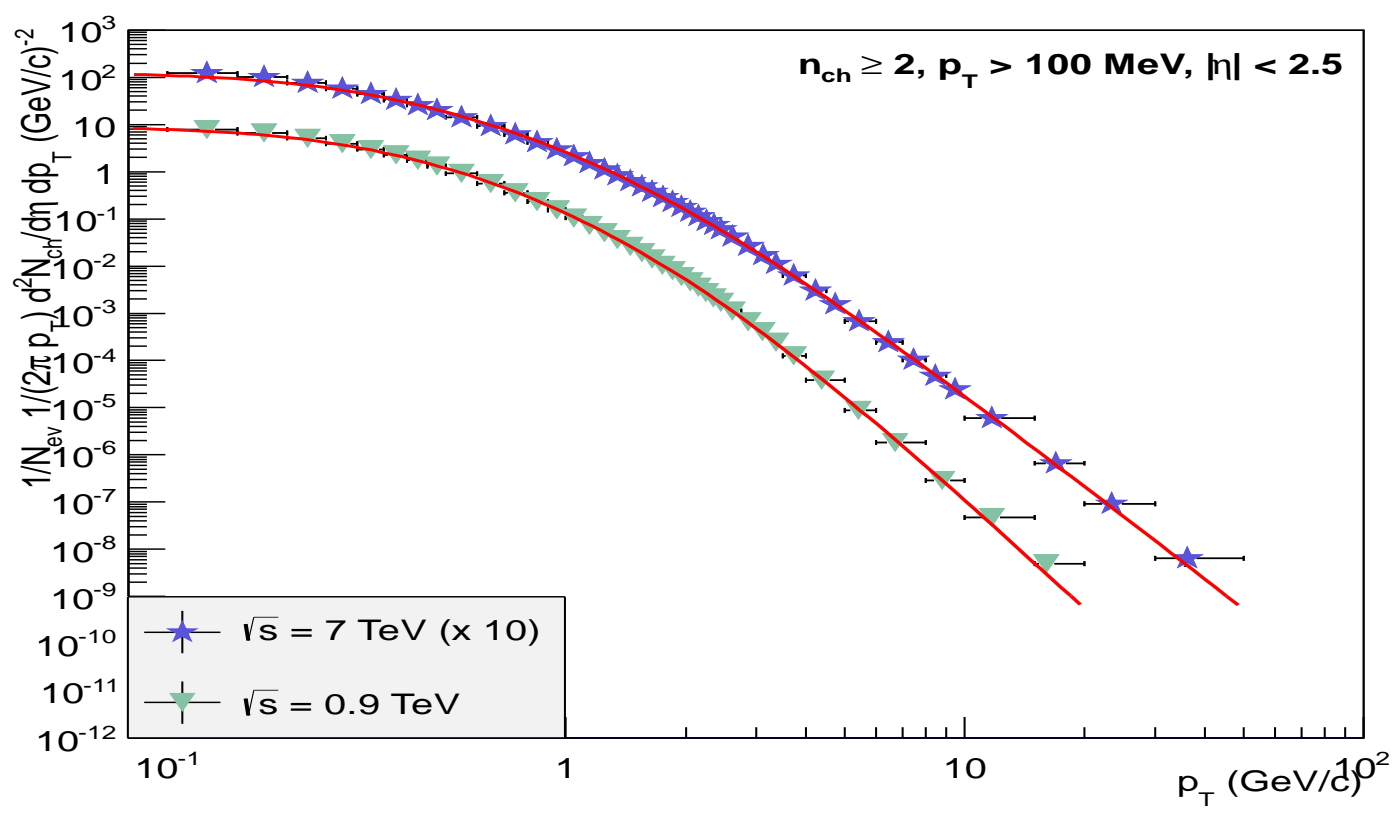

Figure 4. Transverse momentum distributions of charged particles as measured by the ATLAS collaboration in the most inclusive phase-space space region at $\sqrt{s}=0.9$ and $7 \mathrm{TeV}$ together with Tsallis fit. 


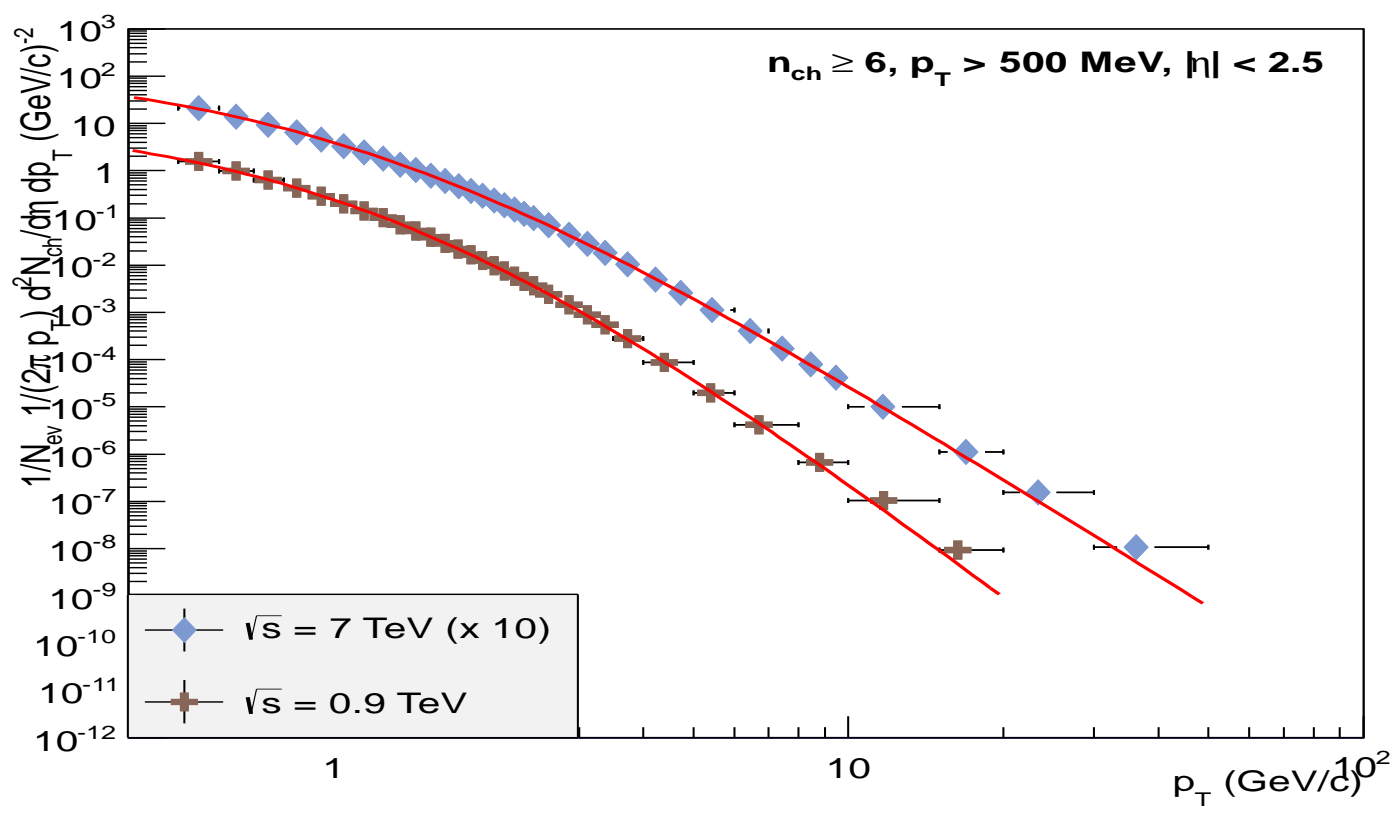

Figure 5. Transverse momentum distributions of charged particles with the smallest contribution from diffractive events as measured by the ATLAS collaboration at $\sqrt{s}=0.9$ and $7 \mathrm{TeV}$ together with Tsallis fit.

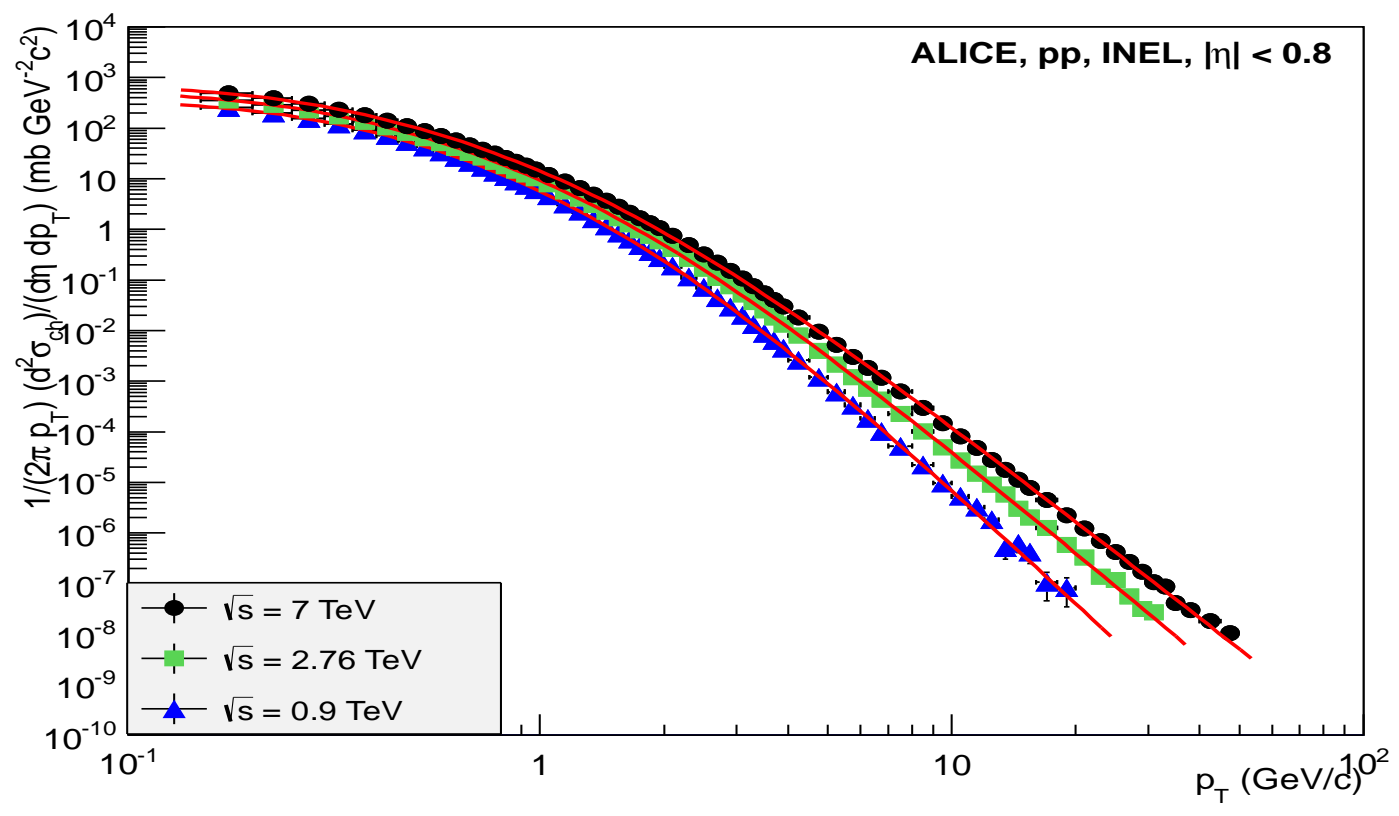

Figure 6. Cross section of charged particles as measured by the ALICE collaboration [24] at different energies. 


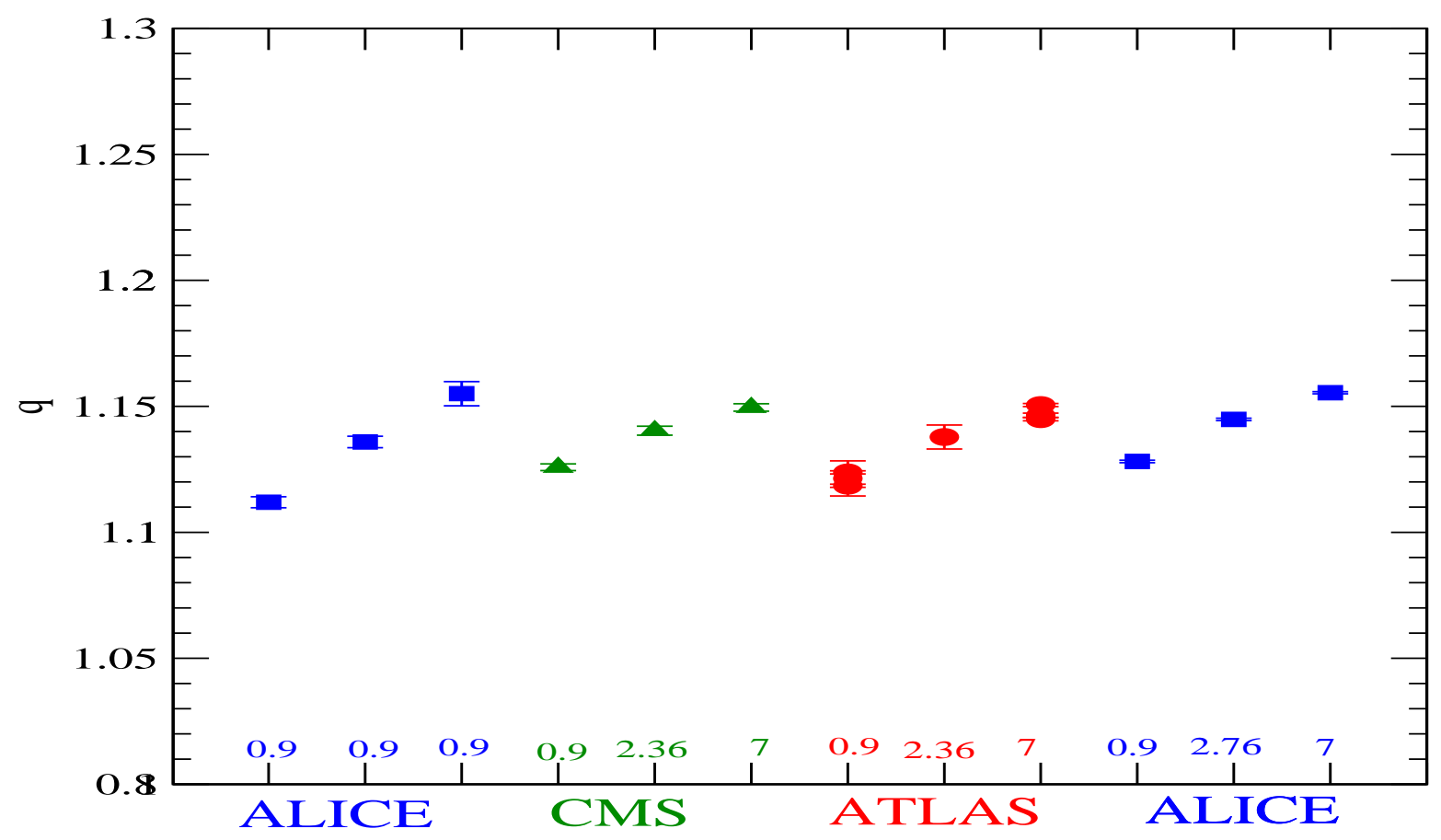

Figure 7. The Tsallis parameter $q$ obtained from fits to the $p_{T}$ spectrum. Results from the ALICE collaboration are indicated with square points, CMS by triangles and ATLAS by circles. The beam energy is given on the $\mathrm{x}$-axis. The first three points from ALICE correspond to different values of accepted charged particles, nacc $=3,7$ and 17 respectively.

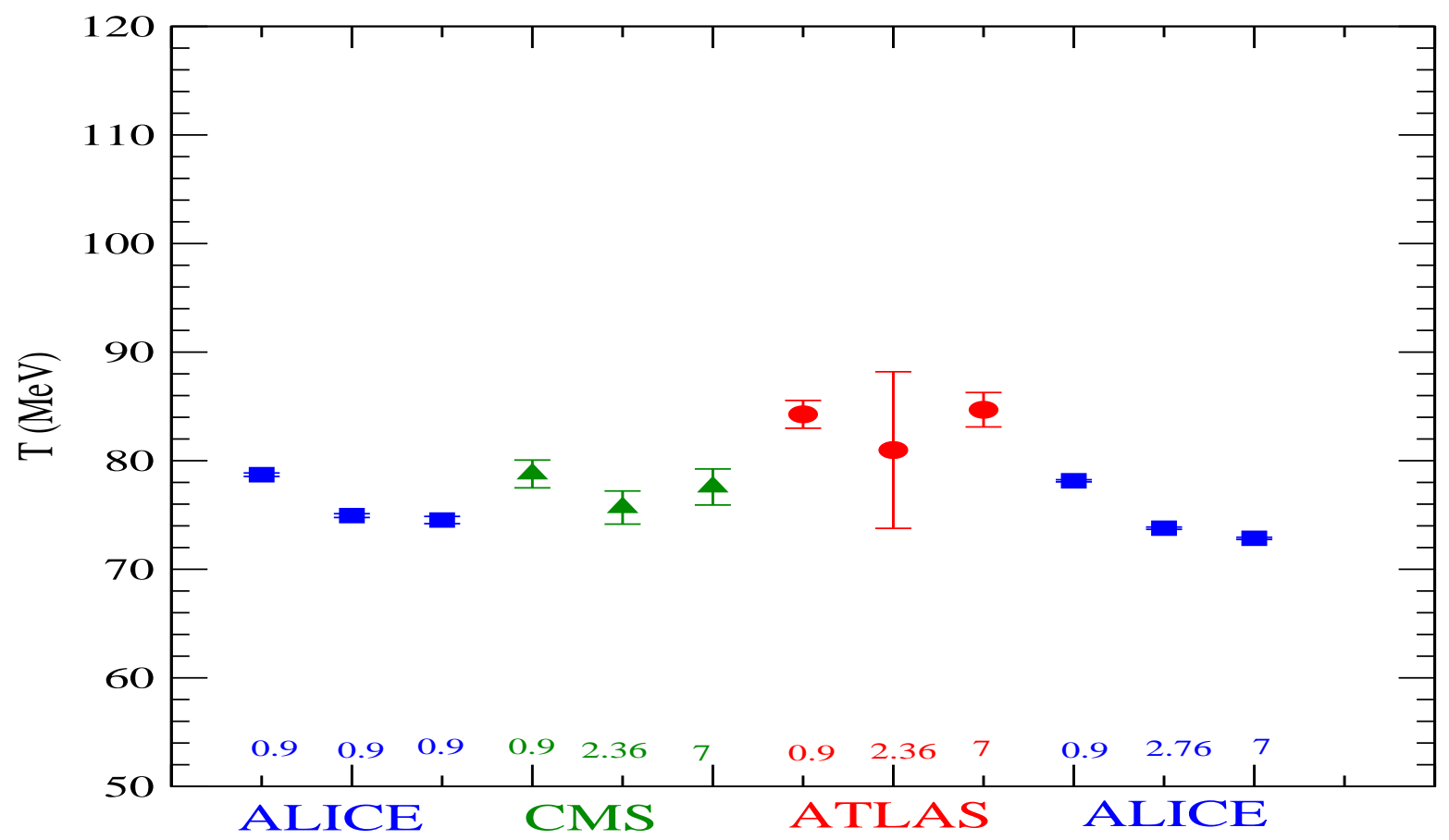

Figure 8. The q-temperature, $T$, obtained from fits to the $p_{T}$ spectrum. Results from the ALICE collaboration are indicated with square points, from CMS by triangles, those from ATLAS by circles. The first three points were obtained at a beam energy of $900 \mathrm{GeV}$ and correspond to different values of accepted charged particles, nacc $=3,7$ and 17 respectively. 


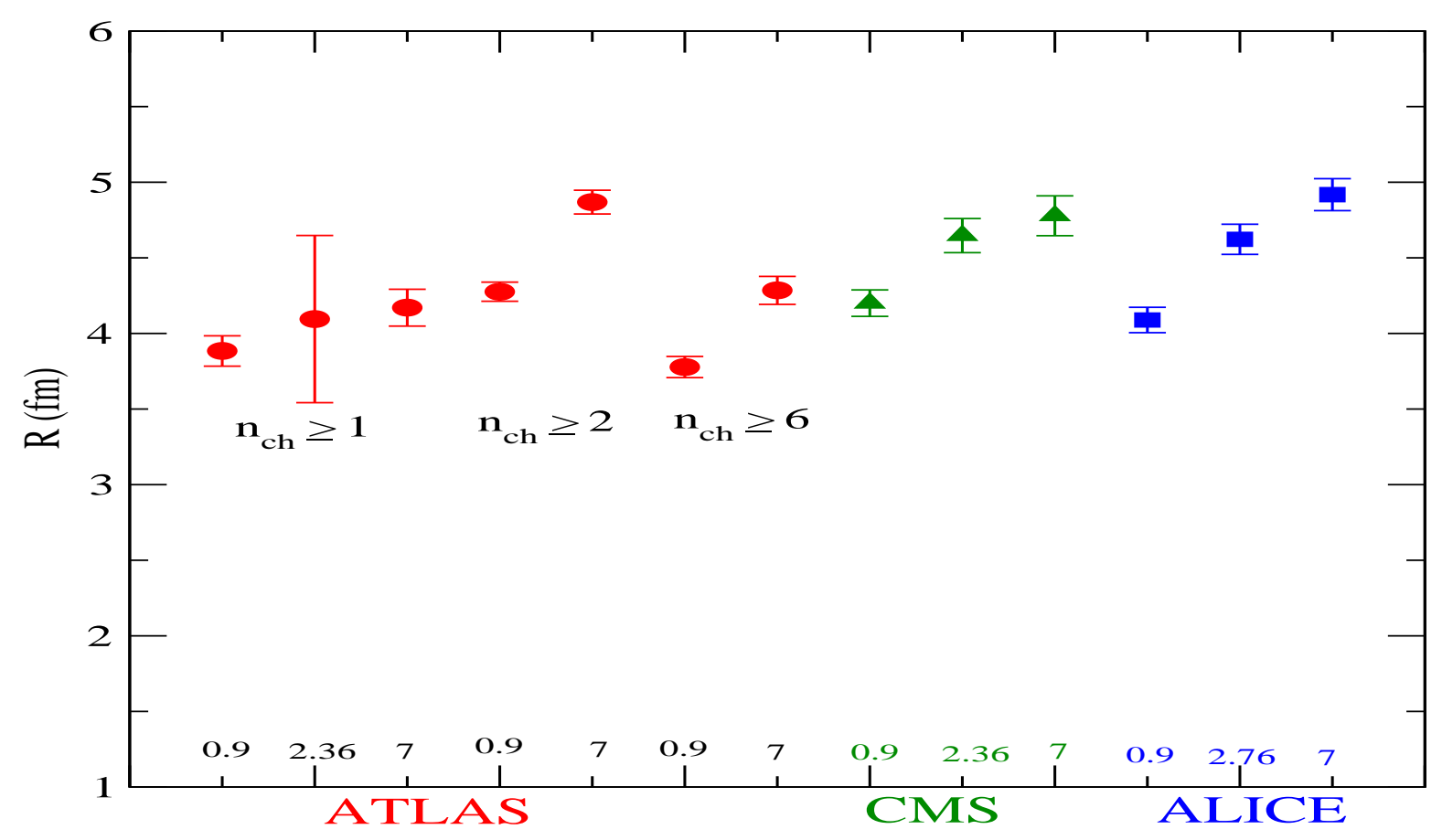

Figure 9. The radius, $R$, obtained from fits to the $p_{T}$ spectrum. The results from ATLAS are indicated by circles, CMS by triangles and ALICE by square points. The ATLAS points correspond to different acceptances of charged particles. The beam energies are given on the $\mathrm{x}$-axis.

\section{Conclusions}

A thermodynamically consistent form of the Tsallis distribution, Eq. (17), gives an excellent description of the transverse momentum spectra and cross section of the primary charged particles measured in $p-p$ collisions at $\sqrt{s}=0.9,2.36,7 \mathrm{TeV}$ and $2.76 \mathrm{TeV}$, respectively.

The values of the Tsallis parameter, $q$, are found between 1.11 to 1.15 and show a clear increase with beam energy.

As observed from the fit of $p_{T}$ spectra measured by the ALICE, ATLAS and CMS collaborations [20, 23, 21, 22, 24], the q-temperature, $T$, is consistent with being constant as a function of beam energy.

The values of radius, $R$, shows a small increase with beam energy.

Overall, the values obtained for the Tsallis parameter, $q$, are remarkably consistent, a feature which does not become apparent when using the parameterization of Eq. (18).

It is concluded that the hadronic system created in high-energy $\mathrm{p}-\mathrm{p}$ collisions at central rapidity can be seen as obeying Tsallis thermodynamics. 


\section{Acknowledgments}

We acknowledge useful discussions with $\mathrm{C}$. Tsallis and M. Floris.

[1] C. Tsallis, J. Statist. Phys. 52, 479 (1988).

[2] T.S. Biró, Physica A 392, 3132 (2013).

[3] C. Tsallis, R. S. Mendes, A. R. Plastino, Physica A 261, 534 (1998).

[4] F. Buyukkilic and D. Demirhan, Phys. Lett. A 181, 24 (1993).

[5] F. Pennini, A. Plastino, and A.R. Plastino, Phys. Lett. A 208, 309 (1995).

[6] A.M. Teweldeberhan, A.R. Plastino, and H.G. Miller, Phys. Lett. A 343, 71 (2005).

[7] J.M. Conroy and H.G. Miller, Phys. Rev. D 78, 054010 (2008).

[8] J.M. Conroy, H.G. Miller and A.R. Plastino, Phys. Lett. A 374, 4581 (2010).

[9] J. Chen, Z. Zhang, G. Su et al., Phys. Lett. A 300, 65 (2002).

[10] J. Cleymans and D. Worku, J. Phys. G 39, 025006 (2012).

[11] J. Cleymans and D. Worku, Eur. Phys. J. A 48, 160 (2012).

[12] T. S. Biró, G. Purcsel and K. Ürmössy, Eur. Phy. J. A 40, 325 (2009).

[13] C.-Y. Wong and G. Wilk, Phys. Rev. D 87, 114007 (2013).

[14] C.-Y. Wong and G. Wilk, Acta Phys. Polon. B 43, 2047 (2012).

[15] I. Sena and A. Deppman, Eur. Phys. J. A 49, 17 (2013).

[16] J. Cleymans, G.I. Lykasov, A.S. Parvan, A.S. Sorin, O.V. Teryaev, D. Worku, Phys. Lett. B 723351 (2013).

[17] B. I. Abelev et al. (STAR collaboration), Phys. Rev. C 75, 064901 (2007).

[18] A. Adare et al. (PHENIX collaboration), Phys. Rev. D 83, 052004, (2011);

[19] A. Adare et al. (PHENIX collaboration), Phys. Rev. C 83, 064903 (2011).

[20] K. Aamodt, et al. (ALICE collaboration), Phys. Lett. B 693, 53 (2010).

[21] V. Khachatryan, et al. (CMS collaboration), JHEP 02, 041 (2010);

[22] V. Khachatryan, et al. (CMS collaboration), Phys. Rev. Lett. 105, 022002 (2010).

[23] G. Aad, et al. (ATLAS collaboration), New J. Phys. 13, 053033 (2011).

[24] K. Aamodt, et al. (ALICE collaboration), Eur. Phys. J. C 73, 2662 (2013).

[25] S. R. deGroot, W. A. van Leeuwen, Ch. van der Weert, Relativistic Kinetic Theory (North Holland, 1980). 